\title{
Research on the Spin-Hamiltonian Parameters and Local Structure for the Tetragonal $\mathrm{Mo}^{5+}$ Centers in $\mathrm{CaWO}_{4}$ Crystal
}

\author{
Y. $\mathrm{MEI}^{a, b}$, W.-C. ZHENG ${ }^{c, *}$, C.-F. WEI ${ }^{a}$ \\ ${ }^{a}$ School of Physics and Electronic Engineering, Mianyang Normal University, Mianyang 621000, P.R. China \\ ${ }^{b}$ Research Center of Computational Physics, Mianyang Normal University, Mianyang 621000, P.R. China \\ ${ }^{c}$ Department of Material Science, Sichuan University, Chengdu 610064, P.R. China
}

(Received January 14, 2014)

The spin-Hamiltonian parameters ( $g$ factors $g_{\|}, g_{\perp}$ and hyperfine structure constants $A_{\|}(\mathrm{A}), A_{\perp}(\mathrm{A}), A_{\|}(\mathrm{B})$ and $A_{\perp}(\mathrm{B}), A(\mathrm{~A})$ and $A(\mathrm{~B})$ belonging to isotopes ${ }^{95} \mathrm{Mo}^{5+}$ and ${ }^{97} \mathrm{Mo}^{5+}$ ) of $\mathrm{Mo}^{5+}$ ion at the tetragonally-compressed tetrahedral $\mathrm{W}^{6+}$ site in $\mathrm{CaWO}_{4}$ crystal are calculated from the high-order perturbation formulae based on the two-mechanism model, where besides the contributions to spin-Hamiltonian parameters due to the crystal-field mechanism concerning the crystal-field excited states in the extensively-applied crystal-field theory, those due to charge-transfer mechanism concerning charge-transfer excited states (which are omitted in crystal-field theory) are included. The calculated results are in reasonable agreement with the experimental values. The calculations show that for the high-valence state $d^{n}$ ions (e. g., $\mathrm{Mo}^{5+}$ considered) in crystals, the contributions due to charge-transfer mechanism should be taken into account in the studies of spin-Hamiltonian parameters. The local structure of $\mathrm{Mo}^{5+}$ center in $\mathrm{CaWO}_{4}$ crystal due to the impurity-induced local lattice relaxation is estimated from the calculations. The results are discussed.

DOI: $10.12693 /$ APhysPolA.126.1275

PACS: $76.30 . \mathrm{He}, 71.70 . \mathrm{Ch}, 75.10 . \mathrm{Dg}$

\section{Introduction}

Tungstates crystals $\mathrm{AWO}_{4}$ (where A indicates a divalent cation) with sheelite-type structure have attracted investigative efforts because they can be readily grown as good-sized, stable, colorlessly transparent and hard crystals. On being doped with rare earth and transition metal ions, they have the potential applications in solid state lasers, luminescence, phosphor and scintillator devices [1-6]. This fact caused a number of spectroscopic studies for $\mathrm{AWO}_{4}$ crystals doped with rare earth and transition metal ions [1-12]. Decades ago, Azarbayejani and Merlo [12] measured the electron paramagnetic resonance (EPR) spectra of $\mathrm{Mo}^{5+}$-doped $\mathrm{CaWO}_{4}$ crystal and found that $\mathrm{Mo}^{5+}$ ion occupies the tetragonallycompressed $\mathrm{W}^{6+}$ site. The spin-Hamiltonian parameters ( $g$ factors $g_{\|}, g_{\perp}$ and hyperfine structure constants $A_{\|}(\mathrm{A}), A_{\perp}(\mathrm{A}), A_{\|}(\mathrm{B})$ and $A_{\perp}(\mathrm{B}), A(\mathrm{~A})$ and $A(\mathrm{~B})$ belong to isotopes ${ }^{95} \mathrm{Mo}^{5+}$ and ${ }^{97} \mathrm{Mo}^{5+}$ ) of this $\mathrm{Mo}^{5+}$ tetrahedral center were reported from the measurement [12]. Up to date there is still lack of the theoretical explanations for these spin-Hamiltonian parameters.

For $d^{n}$ ions in crystals, the spin-Hamiltonian parameters are generally calculated theoretically by the extensively-applied crystal-field (CF) theory where only the contributions due to $\mathrm{CF}$ mechanism concerning the interactions of $\mathrm{CF}$ excited states with the ground state are considered [13-15]. However, strictly speaking,

\footnotetext{
${ }^{*}$ corresponding author; e-mail: zhengwc1@163.com
}

the spin-Hamiltonian parameters originate from two contributions or mechanisms, one is the above $\mathrm{CF}$ mechanism and another is the charge-transfer (CT) mechanism concerning with the interactions of $\mathrm{CT}$ excited states with the ground state [16-18]. The neglect of CT mechanism in CF theory is due to the CT energy levels being often much higher than the CF energy levels [19], which results in the very weak influence of CT energy levels on the ground state. However, since the CT energy levels lower with the increase of the valence state of $d^{n}$ ions [19], for the high-valence state $d^{n}$ ions (e. g., $\mathrm{Mo}^{5+}$ considered here) in crystals, the reasonable and exact calculations of spin-Hamiltonian parameters should also take the contributions due to CT mechanism into account and so the two (CF and CT)-mechanism model should be used. In this paper, we calculate the spinHamiltonian parameters of $\mathrm{Mo}^{5+}$-doped $\mathrm{CaWO}_{4}$ crystals from the high-order perturbation formulae based on the two-mechanism model. In view of the fact that the spinHamiltonian parameters of a paramagnetic ion in crystals depend sensitively upon its immediate environment, the local (or defect) structure of $\mathrm{Mo}^{5+}$ centers caused by the impurity-induced local crystal relaxation in $\mathrm{CaWO}_{4}$ crystals can be estimated from the calculations. The results are discussed.

\section{Calculation}

The one-electron basis functions in the two-mechanism model for a tetrahedral $d^{n}$ cluster can be expressed as the linear combinations of $d$ orbitals $\left|d_{\gamma}\right\rangle$ of $d^{n}$ ions and the $p$ orbitals $\left|\pi_{\gamma}\right\rangle$ and $\left|\sigma_{\gamma}\right\rangle$ of ligand $[17,18]$ : 


$$
\begin{aligned}
& \psi_{\mathrm{e}}^{\chi}=N_{\mathrm{e}}^{\chi}\left(\left|d_{\mathrm{e}}\right\rangle+\sqrt{3} \lambda_{\pi}^{\chi}\left|\pi_{\mathrm{e}}\right\rangle\right), \\
& \psi_{\mathrm{t}}^{\chi}=N_{\mathrm{t}}^{\chi}\left(\mid d_{\mathrm{t}}\left\langle+\lambda_{\sigma}^{\chi}\right| \sigma_{\mathrm{t}}\left\langle+\lambda_{\pi}^{\chi} \mid \pi_{\mathrm{t}}\right\rangle\right),
\end{aligned}
$$

where the subscript $\gamma(=\mathrm{t}$ or e) indicates the irreducible representation of $T_{d}$ group and the superscript $\chi(=\mathrm{a}$ or b) stands for the anti-bonding orbitals related to $\mathrm{CF}$ mechanism and bonding orbitals concerning CT mechanism. $N_{\gamma}$ (normalization coefficients) and $\lambda_{\beta}$ (the orbital mixing coefficients, $\beta=\sigma$ or $\pi$ ) are the molecular orbital (MO) coefficients.

The perturbation formulae of spin-Hamiltonian parameters for $d^{1}$ center in crystals depend upon its ground state and symmetry [13-15]. From the observed $g_{\|}>g_{\perp}$, one can conclude that the ground state of $\mathrm{Mo}^{5+}$ in $\mathrm{CaWO}_{4}$ is $\left|d_{z}^{2}\right\rangle$. Thus, from the above one-electron basis functions and by adding the spin-orbit interaction term $H_{\mathrm{SO}}^{\mathrm{CT}}$, the Zeeman (or magnetic) interaction term $H_{\mathrm{Ze}}^{\mathrm{CT}}$ and the hyperfine interaction term $H_{\mathrm{hf}}^{\mathrm{CT}}$ connected with CT mechanism to the traditional perturbation Hamiltonian in the CF mechanism, the high-order perturbation formulae based on the two-mechanism model for $g$ factors of tetragonal $d^{1}$ tetrahedral cluster with the ground state $\left|d_{z}^{2}\right\rangle$ are derived in Ref. [18] and those for hyperfine structure constants $A_{i}$ are derived here. They are

$$
\begin{aligned}
& g_{\|}=g_{\mathrm{e}}+\Delta g_{\|}^{\mathrm{CF}}+\Delta g_{\|}^{\mathrm{CT}}, \\
& \Delta g_{\|}^{\mathrm{CF}}=\frac{3 \zeta_{\mathrm{CF}}^{\prime 2}\left(k_{\mathrm{CF}}-g_{\mathrm{e}}\right)}{\left(E_{1}^{\mathrm{CF}}\right)^{2}}, \\
& \Delta g_{\|}^{\mathrm{CT}}=\frac{3 \zeta_{\mathrm{CT}}^{2} k_{\mathrm{CT}}^{\prime}}{E_{1}^{\mathrm{CT}} E_{2}^{\mathrm{CT}}}, \\
& g_{\perp}=g_{\mathrm{e}}+\Delta g_{\perp}^{\mathrm{CF}}+\Delta g_{\perp}^{\mathrm{CT}}, \\
& \Delta g_{\perp}^{\mathrm{CF}}=\frac{6 \zeta_{\mathrm{CF}} k_{\mathrm{CF}}^{\prime}}{E_{1}^{\mathrm{CF}}}-\frac{3 \zeta_{\mathrm{CF}}^{\prime}\left(\zeta_{\mathrm{CF}} k_{\mathrm{CF}}^{\prime}+\frac{1}{2} \zeta_{\mathrm{CF}}^{\prime} g_{\mathrm{e}}\right)}{\left(E_{1}^{\mathrm{CF}}\right)^{2}}, \\
& \Delta g_{\perp}^{\mathrm{CT}}=\frac{6 \zeta_{\mathrm{CT}} k_{\mathrm{CT}}}{E_{1}^{\mathrm{CT}}}, \\
& A_{\|}=A_{\|}^{(1)}+A_{\|}^{(2) \mathrm{CF}}+A_{\|}^{(2) \mathrm{CT}}, \\
& A_{\|}^{(1)}=P_{\mathrm{CF}}\left(-\kappa+\frac{4}{7}\right) \text {, } \\
& A_{\|}^{(2) \mathrm{CF}}=P_{\mathrm{CF}}^{\prime}\left[\left(g_{\|}^{\mathrm{CF}}-g_{\mathrm{e}}\right)-\frac{1}{7}\left(g_{\perp}^{\mathrm{CF}}-g_{\mathrm{e}}\right)\right], \\
& A_{\|}^{(2) \mathrm{CT}}=P_{\mathrm{CT}}^{\prime}\left(\frac{3 \zeta_{\mathrm{CT}}^{2} k_{\mathrm{CT}}^{\prime}}{E_{1}^{\mathrm{CT}} E_{2}^{\mathrm{CT}}}\right) \text {, } \\
& A_{\perp}=A_{\perp}^{(1)}+A_{\perp}^{(2) \mathrm{CF}}+A_{\perp}^{(2) \mathrm{CT}}, \\
& A_{\perp}^{(1)}=P_{\mathrm{CF}}\left(-\kappa-\frac{2}{7}\right) \text {, } \\
& A_{\perp}^{(2) \mathrm{CF}}=P_{\mathrm{CF}}^{\prime}\left[\frac{15}{14}\left(g_{\perp}^{\mathrm{CF}}-g_{\mathrm{e}}\right)\right] \text {, } \\
& A_{\perp}^{(2) C T}=P_{\mathrm{CT}}^{\prime}\left(\frac{6 \zeta_{\mathrm{CT}} k_{\mathrm{CT}}}{E_{1}^{\mathrm{CT}}}\right)
\end{aligned}
$$

with the spin-orbit parameters $\zeta, \zeta$, the orbit reduction factors $k, k \prime$ and the dipolar hyperfine structure constants $P, P I$ in CF and CT mechanisms $[17,18]$ :

$$
\begin{aligned}
& \zeta_{\mathrm{CF}}=\left(N_{\mathrm{t}}^{\mathrm{a}}\right)^{2}\left[\zeta_{d}^{0}+\left(\sqrt{2} \lambda_{\pi}^{\mathrm{a}} \lambda_{\sigma}^{\mathrm{a}}-\frac{\lambda_{\pi}^{\mathrm{a} 2}}{2}\right) \zeta_{p}^{0}\right], \\
& \zeta_{\mathrm{CF}}^{\prime}=N_{\mathrm{t}}^{\mathrm{a}} N_{\mathrm{e}}^{\mathrm{a}}\left[\zeta_{d}^{0}+\left(\frac{\lambda_{\pi}^{\mathrm{a}} \lambda_{\sigma}^{\mathrm{a}}}{\sqrt{2}}+\frac{\lambda_{\pi}^{\mathrm{a} 2}}{2}\right) \zeta_{p}^{0}\right], \\
& \zeta_{\mathrm{CT}}=N_{\mathrm{t}}^{\mathrm{a}} N_{\mathrm{t}}^{\mathrm{b}}\left[\zeta_{d}^{0}+\left(\frac{\lambda_{\pi}^{\mathrm{a}} \lambda_{\sigma}^{\mathrm{b}}+\lambda_{\pi}^{\mathrm{b}} \lambda_{\sigma}^{\mathrm{a}}}{\sqrt{2}}-\frac{\lambda_{\pi}^{\mathrm{a}} \lambda_{\pi}^{\mathrm{b}}}{2}\right) \zeta_{p}^{0}\right], \\
& \zeta_{\mathrm{CT}}^{\prime}=N_{\mathrm{e}}^{\mathrm{b}} N_{\mathrm{t}}^{\mathrm{a}}\left[\zeta_{d}^{0}+\left(\frac{\lambda_{\pi}^{\mathrm{a}} \lambda_{\sigma}^{\mathrm{b}}}{\sqrt{2}}+\frac{\lambda_{\pi}^{\mathrm{a}} \lambda_{\pi}^{\mathrm{b}}}{2}\right) \zeta_{p}^{0}\right], \\
& k_{\mathrm{CF}}=\left(N_{\mathrm{t}}^{\mathrm{a}}\right)^{2}\left[1-\frac{\lambda_{\pi}^{\mathrm{a} 2}}{2}+\sqrt{2} \lambda_{\pi}^{\mathrm{a}} \lambda_{\sigma}^{\mathrm{a}}+2 \lambda_{\sigma}^{\mathrm{a}} S_{d p}(\sigma)+2 \lambda_{\pi}^{\mathrm{a}} S_{d p}(\pi)\right] \\
& k_{\mathrm{CF}}^{\prime}=N_{\mathrm{t}}^{\mathrm{a}} N_{\mathrm{e}}^{\mathrm{a}}\left[1+\frac{\lambda_{\pi}^{\mathrm{a} 2}}{2}+\frac{\lambda_{\pi}^{\mathrm{a}} \lambda_{\sigma}^{\mathrm{a}}}{\sqrt{2}}\right. \\
& \left.+4 \lambda_{\pi}^{\mathrm{a}} S_{d p}(\pi)+2 \lambda_{\sigma}^{\mathrm{a}} S_{d p}(\sigma)\right], \\
& k_{\mathrm{CT}}=N_{\mathrm{t}}^{\mathrm{a}} N_{\mathrm{t}}^{\mathrm{b}}\left[1+\left(\frac{\lambda_{\pi}^{\mathrm{a}} \lambda_{\sigma}^{\mathrm{b}}+\lambda_{\pi}^{\mathrm{b}} \lambda_{\sigma}^{\mathrm{a}}}{\sqrt{2}}-\frac{\lambda_{\pi}^{\mathrm{a}} \lambda_{\pi}^{\mathrm{b}}}{2}\right)\right. \\
& \left.+\left(\lambda_{\sigma}^{\mathrm{a}}+\lambda_{\sigma}^{\mathrm{b}}\right) S_{d p}(\sigma)+\left(\lambda_{\pi}^{\mathrm{a}}+\lambda_{\pi}^{\mathrm{b}}\right) S_{d p}(\pi)\right], \\
& k_{\mathrm{CT}}^{\prime}=N_{\mathrm{e}}^{\mathrm{a}} N_{\mathrm{t}}^{\mathrm{b}}\left[1+\left(\frac{\lambda_{\pi}^{\mathrm{a}} \lambda_{\sigma}^{\mathrm{b}}}{\sqrt{2}}-\frac{\lambda_{\pi}^{\mathrm{a}} \lambda_{\pi}^{\mathrm{b}}}{2}\right)\right. \\
& \left.+\lambda_{\sigma}^{\mathrm{b}} S_{d p}(\sigma)+\left(3 \lambda_{\pi}^{\mathrm{a}}+\lambda_{\pi}^{\mathrm{b}}\right) S_{d p}(\pi)\right], \\
& P_{\mathrm{CF}}=\left(N_{\mathrm{t}}^{\mathrm{a}}\right)^{2} P_{0}, \quad P_{\mathrm{CF}}^{\prime}=N_{\mathrm{t}}^{\mathrm{a}} N_{\mathrm{e}}^{\mathrm{a}} P_{0}, \\
& P_{\mathrm{CT}}=N_{\mathrm{t}}^{\mathrm{b}} N_{\mathrm{t}}^{\mathrm{a}} P_{0}, \quad P^{\prime}{ }_{\mathrm{CT}}=N_{\mathrm{e}}^{\mathrm{a}} N_{\mathrm{t}}^{\mathrm{b}} P_{0} .
\end{aligned}
$$

In above formulae, the superscripts and subscripts $\mathrm{CF}$ and CT stand for the parameters in the CF and CT mechanisms. $g_{\mathrm{e}}(\approx 2.0023)$ is the free-electron $g$ value. $\kappa$ is the core polarization constant. $E_{1}^{\mathrm{CF}}$ and $E_{\mathrm{j}}^{\mathrm{CT}}$ are the $\mathrm{CF}$ and CT energy levels. $\zeta_{d}^{0}$ and $\zeta_{p}^{0}$ are the spin-orbit parameters of free $d^{n}$ ion and free ligand. $P_{0}$ is the corresponding parameter of free $d^{n}$ ion. For $\left(\mathrm{MoO}_{4}\right)^{3-}$ clusters under study, we have $\zeta_{d}^{0}\left(M o^{5+}\right) \approx 1030 \mathrm{~cm}^{-1}[14], \zeta_{p}^{0}\left(\mathrm{O}^{2-}\right) \approx$ $150 \mathrm{~cm}^{-1}[20], P_{0}\left({ }^{95} \mathrm{Mo}^{5+}\right) \approx-66.7 \times 10^{-4} \mathrm{~cm}^{-1}$ and $P_{0}\left({ }^{97} \mathrm{Mo}^{5+}\right) \approx-68.2 \times 10^{-4} \mathrm{~cm}^{-1}[21] . S_{d p}(\beta)$ are the group overlap integrals which can be calculated from the Slater-type self-consistent field (SCF) functions [22, 23] with the metal-ligand distance $R$. Since the ionic radius $r_{\mathrm{i}}$ of impurity is unlike the radius $r_{\mathrm{h}}$ of the host ion it replaces, the metal-ligand distance $R$ in the impurity center should differ from the corresponding distance $R_{\mathrm{h}}$ in the host crystal. As an approximation, we estimate the distance $R$ by using the empirical formula $R \approx R_{\mathrm{h}}+\frac{1}{2}\left(r_{\mathrm{i}}-r_{\mathrm{h}}\right)$ [24]. For $\mathrm{Mo}^{5+}$ at the $\mathrm{W}^{6+}$ site of $\mathrm{CaWO}_{4}$ crystal, from $r_{\mathrm{i}}\left(\mathrm{Mo}^{5+}\right) \approx 0.60 \AA$, $r_{\mathrm{h}}\left(\mathrm{W}^{6+}\right) \approx 0.56 \AA[25]$ and $R_{\mathrm{h}} \approx 1.782 \AA[26]$, we obtain $R \approx 1.802 \AA$. Thus, we have $S_{d p}(\pi) \approx 0.03320$ and $S_{d p}(\sigma) \approx-0.10744$.

The MO coefficients $N_{\gamma}^{\chi}$ and $\lambda_{\beta}^{\chi}$ needed for the calculations of the parameters in Eq. (3) can be related by the normalization correlations

$$
\begin{aligned}
& N_{\mathrm{e}}^{\chi}=\left[1+3\left(\lambda_{\sigma}^{\chi}\right)^{2}+6 \lambda_{\pi}^{\chi} S_{d p}(\pi)\right]^{-\frac{1}{2}}, \\
& N_{\mathrm{t}}^{\chi}=\left[1+\left(\lambda_{\sigma}^{\chi}\right)^{2}+\left(\lambda_{\pi}^{\chi}\right)^{2}+2 \lambda_{\sigma}^{\chi} S_{d p}(\sigma)+2 \lambda_{\pi}^{\chi} S_{d p}(\pi)\right]^{-\frac{1}{2}}
\end{aligned}
$$




$$
\begin{aligned}
\lambda_{\pi}^{\mathrm{b}} & =-\frac{1+3 \lambda_{\pi}^{\mathrm{a}} S_{d p}(\pi)}{3\left[\lambda_{\pi}^{\mathrm{a}}+S_{d p}(\pi)\right]}, \\
\lambda_{\sigma}^{\mathrm{b}} & =-\frac{1+\lambda_{\pi}^{\mathrm{a}} \lambda_{\pi}^{\mathrm{b}}+\left(\lambda_{\pi}^{\mathrm{a}}+\lambda_{\pi}^{\mathrm{b}}\right) S_{d p}(\pi)+\lambda_{\sigma}^{\mathrm{a}} S_{d p}(\sigma)}{\lambda_{\sigma}^{\mathrm{a}}+S_{d p}(\sigma)}
\end{aligned}
$$

and the approximate relationships

$$
\begin{aligned}
& f_{\mathrm{e}}=\left(N_{\mathrm{e}}^{\mathrm{a}}\right)^{4}\left[1+6 \lambda_{\pi}^{\mathrm{a}} S_{d p}(\pi)+9\left(\lambda_{\pi}^{\mathrm{a}}\right)^{2} S_{d p}^{2}(\pi)\right] \\
& f_{\mathrm{t}}=\left(N_{\mathrm{t}}^{\mathrm{a}}\right)^{4}\left[1+2 \lambda_{\sigma}^{\mathrm{a}} S_{d p}(\sigma)+2 \lambda_{\pi}^{\mathrm{a}} S_{d p}(\pi)\right. \\
& \left.\quad+2 \lambda_{\sigma}^{\mathrm{a}} S_{d p}(\sigma) \lambda_{\pi}^{\mathrm{a}} S_{d p}(\pi)+\left(\lambda_{\sigma}^{\mathrm{a}}\right)^{2} S_{d p}^{2}(\sigma)+\left(\lambda_{\sigma}^{\mathrm{a}}\right)^{2} S_{d p}^{2}(\sigma)\right],(6
\end{aligned}
$$
in which we assume the covalence factor $f_{\mathrm{t}} \approx f_{\mathrm{e}} \approx f_{\gamma}$ for decreasing the number of adjustable parameter and take $f_{\gamma}$ as an adjustable parameter.

The CT energy levels of $\left(\mathrm{MoO}_{4}\right)^{3-}$ clusters have not been reported. Since the CT energy levels of the $\left(\mathrm{CuCl}_{4}\right)^{2-}$ tetrahedral clusters are near to those of the $\left(\mathrm{CuCl}_{6}\right)^{4-}$ octahedral clusters [27, 28], for $\left(\mathrm{MoO}_{4}\right)^{3-}$ tetrahedral clusters, we approximately take $E_{1}^{\mathrm{CT}} \approx$ $32400 \mathrm{~cm}^{-1}$ and $E_{2}^{\mathrm{CT}} \approx 36400 \mathrm{~cm}^{-1}$, the values of $\left(\mathrm{MoO}_{6}\right)^{7-}$ octahedral clusters [29].

In the $\mathrm{CF}$ theory, the $\mathrm{CF}$ energy level

$$
E_{1}^{\mathrm{CF}}=E\left(\left|d_{x y, y z}\right\rangle\right)-E\left(\left|d_{z}\right\rangle\right) \approx 10 D q-D s-10 D t,
$$

where the tetragonal field parameters $D s$ and $D t$ can be calculated from the superposition model [30]. In the model, they can be written as

$$
\begin{aligned}
& D s=-\frac{1}{7} B_{20}=-\frac{4}{7} \bar{A}_{2}(R)\left(3 \cos ^{2} \theta-1\right) \\
& \begin{aligned}
D t & =-\frac{1}{21}\left(B_{40}-\frac{\sqrt{70}}{5} B_{44}\right)= \\
& -\frac{4}{21} \bar{A}_{4}(R)\left(35 \cos ^{4} \theta-30 \cos ^{2} \theta+3+7 \sin ^{4} \theta\right),
\end{aligned}
\end{aligned}
$$

where $B_{k l}$ are the $\mathrm{CF}$ parameters in Wybourne notation $[31,32] . \quad \bar{A}_{k}(R)(k=2,4)$ are the intrinsic parameters. For $4 d^{n}$ ions in crystals, the ratio $\bar{A}_{2}(R) / \bar{A}_{4}(R) \approx 6 \pm 2$ was found [29, 33-35], we take the average value $\bar{A}_{2}(R) / \bar{A}_{4}(R) \approx 6$ here. The parameter $\bar{A}_{4}(R)$ for $d^{n}$ tetrahedral cluster can take the form $\bar{A}_{4}(R) \approx-\frac{27}{16} D q[36]$, where $D q$ is the cubic field parameter. The value of $D q$ for $\left(\mathrm{MoO}_{4}\right)^{3-}$ cluster in crystals has not been reported and is estimated approximately as follows.

The optical spectral data for various $d^{n}$ ions in crystals suggest that the value of $D q$ for $4 d^{n}$ clusters is about 1.5(1) times that of the isoelectronic $3 d^{n}$ clusters [19]. So, from $D q \approx 1350(50) \mathrm{cm}^{-1}$ of the $3 d^{1}\left(\mathrm{CrO}_{4}\right)^{3-}$ tetrahedral clusters [37], we obtain for the corresponding $4 d^{1}\left(\mathrm{MoO}_{4}\right)^{3-}$ tetrahedral cluster, $D q \approx 1900 \mathrm{~cm}^{-1}$. $\theta$ refers to the angle between the metal-ligand distance $R$ and $\mathrm{C}_{4}$ axis. Analogous to the bonding length $R$, the bonding angle $\theta$ in the impurity center may be different from the corresponding angle $\theta_{\mathrm{h}}$ in the host crystal. We assume $\theta \approx \theta_{\mathrm{h}}+\Delta \theta$, where $\theta_{\mathrm{h}} \approx 56.89^{\circ}[26]$ in the host $\mathrm{CaWO}_{4}$ crystal and $\Delta \theta$ represents the impurityinduced angular distortion. $\Delta \theta$ is also treated as an adjustable parameter. Thus, in the above formulae, we have three parameters $f_{\gamma}, \Delta \theta$ and $\kappa$ left as the adjustable parameters. From the calculated (with the above high-order perturbation formulae)-to-experimental fitting of spin-Hamiltonian parameters for $\mathrm{CaWO}_{4}$ : $\mathrm{Mo}^{5+}$, we obtain

$$
f_{\gamma} \approx 0.525, \quad \Delta \theta \approx 0.4^{\circ}, \quad \kappa \approx 0.41 .
$$

The MO coefficients based on the value of $f_{\gamma}$ are given in Table I. The parameters in Eq. (3) calculated from these MO coefficients are listed in Table II. The calculated spin-Hamiltonian parameters are compared with the experimental values in Table III.

\section{TABLE I}

The molecular orbital (MO) coefficients for $\left(\mathrm{MoO}_{4}\right)^{3-}$ tetrahedral clusters in $\mathrm{CaWO}_{4}$ : $\mathrm{Mo}^{5+}$ crystal.

\begin{tabular}{c|c|c|c|c|c|c|c}
\hline \hline$N_{\mathrm{t}}^{\mathrm{a}}$ & $N_{\mathrm{e}}^{\mathrm{a}}$ & $N_{\mathrm{t}}^{\mathrm{b}}$ & $N_{\mathrm{e}}^{\mathrm{b}}$ & $\lambda_{\sigma}^{\mathrm{a}}$ & $\lambda_{\pi}^{\mathrm{a}}$ & $\lambda_{\sigma}^{\mathrm{b}}$ & $\lambda_{\pi}^{\mathrm{b}}$ \\
\hline 0.8767 & 0.8745 & 0.3622 & 0.2350 & 0.3709 & -0.5274 & -2.3792 & 0.6391
\end{tabular}

TABLE II

The spin-orbit parameters $\zeta, \zeta^{\prime}\left(\mathrm{cm}^{-1}\right)$, the orbital reduction factors $k, k \prime$ and the dipolar hyperfine structure constants $P, P^{\prime}$ (in $\left.10^{-4} \mathrm{~cm}^{-1}\right)$ in $\mathrm{CF}$ and $\mathrm{CT}$ mechanisms for $\left(\mathrm{MoO}_{4}\right)^{3-}$ tetrahedral clusters in $\mathrm{CaWO}_{4}: \mathrm{Mo}^{5+}$ crystal.

\begin{tabular}{c|c|c|c|c|c|c|c}
\hline \hline$\zeta_{\mathrm{CF}}$ & $\zeta^{\prime}{ }_{\mathrm{CF}}$ & $\zeta_{\mathrm{CT}}$ & $\zeta_{\mathrm{CT}}$ & $k_{\mathrm{CF}}$ & $k_{\mathrm{CF}}$ & $k_{\mathrm{CT}}$ & $k \prime_{\mathrm{CT}}$ \\
743.8 & 789.8 & 250.0 & 360.3 & 0.3610 & 0.4698 & 0.7757 & 0.7222 \\
\hline$P_{\mathrm{CF}}(\mathrm{A})^{a}$ & $P{ }_{\mathrm{CF}}(\mathrm{A})^{a}$ & $P_{\mathrm{CT}}(\mathrm{A})^{a}$ & $P^{\prime}{ }_{\mathrm{CT}}(\mathrm{A})^{a}$ & $P_{\mathrm{CF}}(\mathrm{B})^{a}$ & $P^{\prime}{ }_{\mathrm{CF}}(\mathrm{B})^{a}$ & $P_{\mathrm{CT}}(\mathrm{B})^{a}$ & $P^{\prime} \mathrm{CT}(\mathrm{B})^{a}$ \\
-51.3 & -51.1 & -21.2 & -21.1 & -52.4 & -52.3 & -21.7 & -21.6 \\
\hline
\end{tabular}

\footnotetext{
${ }^{a} P(\mathrm{~A})$ and $P(\mathrm{~B})$ belong to isotopes ${ }^{95} \mathrm{Mo}^{5+}$ and ${ }^{97} \mathrm{Mo}^{5+}$.
} 
The spin-Hamiltonian parameters ( $g$ factor $g_{\|}, g_{\perp}$ and hyperfine structure constants $A_{\|}(\mathrm{A}), A_{\perp}(\mathrm{A}), A_{\|}(\mathrm{B})$ and $A_{\perp}(\mathrm{B}), A(\mathrm{~A})$ and $A(\mathrm{~B})$ belong to isotopes ${ }^{95} \mathrm{Mo}^{5+}$ and ${ }^{97} \mathrm{Mo}^{5+}$, constants $A$ are in units of $\left.10^{-4} \mathrm{~cm}^{-1}\right)$ for the tetragonal $\left(\mathrm{MoO}_{4}\right)^{3-}$ clusters in $\mathrm{CaWO}_{4}$ crystals.

\begin{tabular}{|c|c|c|c|c|}
\hline $\begin{array}{c}\Delta g_{\|}^{\mathrm{CF}} \\
-0.0175\end{array}$ & $\begin{array}{c}\Delta g_{\|}^{\mathrm{CT}} \\
0.0001\end{array}$ & $\begin{array}{c}g_{\|}(\text {calc. }) \\
1.9849\end{array}$ & $\begin{array}{c}g_{\|}(\text {expt. [12] }) \\
1.987\end{array}$ & \\
\hline$\Delta g_{\perp}^{\mathrm{CF}}$ & $\Delta g_{\perp}^{\mathrm{CT}}$ & $g_{\perp}($ calc. $)$ & $g_{\perp}($ expt. [12]) & \\
\hline-0.1508 & 0.0359 & 1.8874 & 1.887 & \\
\hline$A_{\|}^{(1)}(\mathrm{A})$ & $A_{\|}^{(2) \mathrm{CF}}(\mathrm{A})$ & $A_{\|}^{(2) \mathrm{CT}}(\mathrm{A})$ & $A_{\|}(\mathrm{A})($ calc. $)$ & $A_{\|}(\mathrm{A})(\operatorname{expt} .[12])$ \\
\hline-8.27 & -0.21 & -0.002 & -8.48 & $8.39^{a}$ \\
\hline$A_{\perp}^{(1)}(\mathrm{A})$ & $A_{\perp}^{(2) \mathrm{CF}}(\mathrm{A})$ & $A_{\perp}^{(2) \mathrm{CT}}(\mathrm{A})$ & $A_{\perp}(\mathrm{A})($ calc. $)$ & $A_{\perp}(\mathrm{A})(\operatorname{expt} .[12])$ \\
\hline 35.66 & 8.26 & -0.76 & 43.16 & $41.18^{a}$ \\
\hline$A_{\|}^{(1)}(\mathrm{B})$ & $A_{\|}^{(2) \mathrm{CF}}(\mathrm{B})$ & $A_{\|}^{(2) \mathrm{CT}}(\mathrm{B})$ & $A_{\|}(\mathrm{B})($ calc. $)$ & $A_{\|}(\mathrm{B})($ expt.[12] $)$ \\
\hline-8.45 & -0.21 & -0.002 & -8.66 & $8.64^{a}$ \\
\hline$A_{\perp}^{(1)}(\mathrm{B})$ & $A_{\perp}^{(2) C F}(\mathrm{~B})$ & $A_{\perp}^{(2) C T}(\mathrm{~B})$ & $A_{\perp}(\mathrm{B})($ calc. $)$ & $A_{\perp}(\mathrm{B})(\operatorname{expt} .[12])$ \\
\hline 36.46 & 8.44 & -0.78 & 44.12 & $42.52^{a}$ \\
\hline
\end{tabular}

${ }^{a}$ The values are actually the absolute values.

\section{Discussion}

The signs of hyperfine structure constants $A_{i}$ are hard to be determined solely by EPR experiment [14, 21, 38]. So, even though the values of $A_{i}$ are frequently written as positive in EPR experiment, they are actually the absolute values. For $\mathrm{Mo}^{5+}$ ions in $\mathrm{CaWO}_{4}$ crystal, our calculations suggest that $A_{\|}$is negative and $A_{\perp}$ is positive (see Table III).

The angular distortion $\Delta \theta \neq 0$ confirms the expectation that the bonding angle $\theta$ in the $\mathrm{Mo}^{5+}$ impurity center in $\mathrm{CaWO}_{4}$ is different from the corresponding angle $\theta_{h}$ in the host crystal because of the impurity-induced local lattice relaxation. So, the local structure of a paramagnetic impurity center in crystals can be acquired by studying its EPR data.

Table III shows that by using three adjustable parameters, the calculated spin-Hamiltonian parameters $g_{\|}$, $g_{\perp}, A_{\|}(\mathrm{A}), A_{\perp}(\mathrm{A}), A_{\|}(\mathrm{B})$ and $A_{\perp}(\mathrm{B})$ are in reasonable agreement with the experimental values. This suggests that the high-order perturbation formulae based on the two-mechanism model are effective here. To characterize the relative importance of CT mechanism, we introduce the ratio $\left|Q^{\mathrm{CT}} / Q^{\mathrm{CF}}\right|$. From Table III, we obtain $\left|Q^{\mathrm{CT}} / Q^{\mathrm{CF}}\right| \approx 0.6 \%, 24 \%, 1 \%$ and $9 \%$ for $Q=\Delta g_{\|}, \Delta g_{\perp}$, $A_{\|}^{(2)}$ and $A_{\perp}^{(2)}$. It can be seen that the values $\left|Q^{\mathrm{CT}} / Q^{\mathrm{CF}}\right|$ of relative importance of CT mechanism for $\Delta g_{\|}$and $A_{\|}^{(2)}$ are much smaller than those for $\Delta g_{\perp}$ and $A_{\perp}^{(2)}$. The main reason of the above great difference in $\left|Q^{\mathrm{CT}} / Q^{\mathrm{CF}}\right|$ may be due to the contributions to $\Delta g_{\|}$and $A_{\|}^{(2)}$ in both the $\mathrm{CF}$ and CT mechanisms depending upon the third-order perturbation terms [note: the second-order terms are absent according to the derivation, see Eq. (2)], whereas those to $\Delta g_{\perp}$ and $A_{\perp}^{(2)}$ mainly from the second-order perturbation terms. This leads the ratios $\left|Q^{\mathrm{CT}} / Q^{\mathrm{CF}}\right|$ for $\Delta g_{\|}$and $A_{\|}^{(2)}$ to be connected roughly with $\left(E^{\mathrm{CT}} / E^{\mathrm{CF}}\right)^{2}$, but those for $\Delta g_{\perp}$ and $A^{(2)}$ roughly with $E^{\mathrm{CT}} / E^{\mathrm{CF}}$ [see Eq. (2)]. Since $E^{\mathrm{CT}} / E^{\mathrm{CF}}<0.5$ (note: the calculated $E_{1}^{\mathrm{CF}} \approx 13240 \mathrm{~cm}^{-1}$ here), the above large difference in the relative importance can be understood. In consideration of the great relative importance of CT mechanism to $\Delta g_{\perp}$ and $A_{\perp}^{(2)}$, in the rational and precise calculations of spin-Hamiltonian parameters and the estimations of the local structure of impurity centers (by analyzing the spin-Hamiltonian parameters) for the high-valence state $d^{n}$ ions in crystals, one should apply the method based on the two-mechanism model.

\section{Acknowledgments}

This project is supported by the Initial Foundation of Mianyang Normal University (Grant No. MQD2011A05), the Key Project of Sichuan Provincial Science and Technology Department (Grant No. 2012SZZ025, 2012JY0045).

\section{References}

[1] A.A. Kaminskii, Laser Crystals: Their Physics and Properties, Springer-Verlag, Berlin 1981.

[2] J.Y.Sun, Y.N. Sun, C. Cao, Z.G. Xia, H.Y. Du, Appl. Phys. B 111, 367 (2013).

[3] K.G. Sharma, N.R. Singh, J. Rare Earths 30, 310 (2012).

[4] J.S. Liao, B. Qiu, H.R. Wen, J.L. Chen, W.X. You, L.B. Liu, J. Alloys Comp. 487, 758 (2009).

[5] V.V. Laguta, A. Vedda, D.Di. Martino, M. Martino, M. Nikl, E. Mihokova, J. Rosa, Y. Usuki, Phys. Rev. $B$ 71, 235108 (2005). 
[6] E. Baibekov, I. Kurkin, M. Gufurov, B. Endeward, R. Rakhmatullin, G. Mamin, J. Magn. Reson. 209, 61 (2011).

[7] C.A. Morrison, R.P. Leavitt, in: Handbook on the Physics and Chemistry of Rare Earths, Eds. K.A. Gschneidner, Jr., L. Eyring, Vol. 5, NorthHolland Publishing Company, Amsterdam 1982, Chapter 46.

[8] I. Trabelsi, M. Dammak, R. Maalej, M. Kamoun, Physica B 406, 315 (2011)

[9] S. Mahlik, E. Cavalli, M. Bettinelli, M. Grinberg, Radiat. Meas. 56, 1 (2013).

[10] T.H. Yeom, J. Korean. Phys. Soc. 47, 681 (2005).

[11] Z. Sroubek, K. Zdansky, J. Chem. Phys. 44, 3078 (1966).

[12] G.H. Azarbayejani, A.L. Merlo, Phys. Rev. 137, A489 (1965).

[13] J.S. Griffith, The Theory of Transition-Metal Ions, Cambridge University Press, London 1964.

[14] A. Abragam, B. Bleaney, Electron Paramagnetic Resonance of Transition Ions, Oxford University Press, London 1970.

[15] J.R. Pilbrow, Transition Ion Electron Paramagnetic Resonance, Clarendon Press, Oxford 1990.

[16] J.A. Arambura, M. Moreno, Solid State Commun. 62, 513 (1987).

[17] W.C. Zheng, W.Q. Yang, Y. Mei, Mol. Phys. 107, 2245 (2009)

[18] Y. Mei, W.C. Zheng, Y.G. Yang, H.G. Liu, Physica B 407, 4365 (2012).

[19] A.B.P. Lever, Inorganic Electronic Spectroscopy, Elsevier, Amsterdam 1984.

[20] M.L. Du, C. Rudowicz, Phys. Rev. B 46, 8974 (1992).

[21] B.R. McGarvey, J. Phys. Chem. 71, 51 (1967).
[22] E. Clementi, D.L. Raimondi, J. Chem. Phys. 38, 2686 (1963).

[23] E. Clementi, D.L. Raimondi, W.P. Reinhardt, J. Chem. Phys. 47, 1300 (1967).

[24] W.C. Zheng, Physica B 215, 255 (1995).

[25] R.D. Shannon, Acta Crystallogr. A 32, 751 (1976).

[26] R.M. Hazen, L.W. Finger, J.W.E. Mariathasan, J. Phys. Chem. Solids 46, 253 (1985).

[27] R. Valiente, F. Rodriguez, M.T. Barriuso, C. Sousa, C. de Graaf, T.A. Arambura, M. Moreno, High Press. Res. 22, 475 (2002).

[28] J.A. Arambura, M. Moreno, A. Bencini, Chem. Phys. Lett. 140, 462 (1987).

[29] Y. Mei, W.C. Zheng, H.G. Liu, Physica B 430, 27 (2013).

[30] D.J. Newman, B. Ng, Rep. Prog. Phys. 52, 699 (1989).

[31] B.G. Wybourne, Spectroscopic Properties of Rare Earth, Wiley, New York 1965.

[32] Z.Y. Yang, Y. Hao, C. Rudowicz, Y.Y. Yeung, J. Phys. Condens. Matter 16, 348 (2004).

[33] W.C. Zheng, W. Fang, Y. Mei, J. Appl. Phys. 101, 053911 (2007).

[34] W.L. Feng, W.Q. Yang, W.C. Zheng, X.M. Li, J. Alloys Comp. 507, 498 (2010).

[35] W.C. Zheng, Y. Mei, W.Q. Yang, Philos. Mag. 28, 1621 (2009)

[36] W.L. Yu, M.G. Zhao, Phys. Rev. B 37, 9254 (1988).

[37] M. Atanasov, T.C. Brunold, H.U. Gudel, C. Daul, Inorg. Chem. 37, 4589 (1998).

[38] V. Havlicek, P. Novak, B.V. Mill, Phys. Status Solidi B 64, K19 (1974). 